\section{Novel I202N Mutation in CMT1X Gene}

Swathi Beladakere Ramaswamy, MD; Sachin M. Bhagavan, MD; Raghav Govindarajan, MD

Department of Neurology, University of Missouri

\section{ABSTRACT}

This case report describes a novel I202N mutation in the gap junction protein beta 1 (GJ $\beta 1$ ) gene consistent with diagnosis of Charcot-Marie Tooth disease (CMT) in a 63-year-old female who presented with slowly progressive bilateral foot weakness and foot drop over four decades. The discussion emphasizes the novel mutation and the gender difference in severity.

\section{Introduction}

Charcot Marie Tooth disease (CMT) is a heterogeneous group of hereditary motor sensory neuropathies and is the most common inherited neurological condition. ${ }^{1}$ It consists of a spectrum of disorders caused by mutations in various genes whose protein products are expressed in myelin and/or axonal structures within peripheral nerves. ${ }^{1}$ More than 40 causal genes have been identified. Inheritance is variable and includes autosomal recessive and dominant, X-linked, and mitochondrial inheritance patterns., ${ }^{1,2}$ X-linked Charcot-Marie-Tooth disease (CMTX) is the second common genetic variant of CMT. CMTX type 1 causes $90 \%$ of CMTX. ${ }^{3}$ Though the exact mechanisms by which GJBl mutations lead to the CMTIX phenotype are not fully defined, hundereds of pathological mutations have been identified. ${ }^{3}$ The following case discusses one such novel mutation.

\section{Case Report}

A 63-year-old female was referred to the neurology clinic for evaluation of slowly progressive weakness and foot drop. She developed bilateral foot weakness in her teenage years in conjunction with pes cavus and hammer toe deformities. She was able to walk without significant impairment at that time. Over approximately four decades leading up to the time of referral, she experienced worsening foot drop and gait difficulties functionally manifesting as stumbling and tripping. During this time, she also developed gradual weakness in her hands that interfered with writing.

Relevant past medical history at the time of presentation included nine years prior breast cancer treated with lumpectomy, chemotherapy (5-fluorouracil, cyclophosphamide, methotrexate) and radiation therapy, essential tremor, hypertension, hyperlipidemia, cataracts, prediabetes, depression, and corrective surgery for hammer toe deformities. There was no history of alcohol or illicit drug abuse.

The patient has three sons, one of whom was affected similarly although beginning at an earlier age with increased severity and speed of progression. Her two other children did not have medical problems. The patient has three siblings - one brother with a history of tremor, otherwise no relatives with neurologic disease.

Neurological exam was remarkable for significant atrophy of the small hand muscles with more prominent wasting of thenar than hypothenar groups, consistent with 'split hand sign.' Atrophy of bilateral intrinsic foot muscles and pes cavus was present. Muscle strength was significantly diminished as $0 / 5$ bilateral toe extension, $2 / 5$ bilateral foot dorsiflexion and 3/5 intrinsic hand muscles. Light touch and pinprick sensation were also impaired in the hands and feet bilaterally. Vibration was impaired at the fingers and absent at the ankle and toes. Proprioception was decreased in the toes. Deep tendon reflexes were absent at the ankle and biceps, diminished to a lesser degree in the triceps, and normal in the knees. Circumduction of gait was noted bilaterally secondary to foot drop, and the patient was unable to walk on her heels. There were postural and action tremors of the hands. Cranial nerves II-XII were intact. Coordination testing was unremarkable.

Needle EMG and nerve conduction studies (NCS) showed a reduction in amplitude of median and tibial nerves with marked velocity reduction in distal extremities. There were also prolonged latencies in median and tibial motor NCSs. Sensory NCS demonstrated a marked decrease in amplitude and decreased to borderline velocities. EMG of tibialis anterior and gastrocnemius muscles showed mild active denervation and prominent chronic reinnervation of the right extensor digitorum brevis, tibialis anterior, and gastrocnemius (medial head). These findings supported a demyelinating neuropathy with secondary axonal change and axonal sensorimotor polyneuropathy.

This patient presented with progressive, distal weakness with a positive family history of similar symptoms in her son raising the suspicion for hereditary neuropathy. In the setting of possible diagnosis of CMT, focused genetic testing for disease-producing mutations was performed in both the patient and her son, consistent with American Academy of Neurology evidence-based practice parameters for the evaluation of distal symmetric polyneuropathy [5]. Genetic testing performed through GeneDx revealed a novel heterozygous $\mathrm{T}$ to $\mathrm{A}$ transversion (c.605 $\mathrm{T}>\mathrm{A}$ ) in exon two of the GJB1 gene encoding connexon 32 resulting in p.Ile202Asn amino acid substitution predicted to be pathogenic. The patient's son was hemizygous for the same mutation. 


\section{Discussion}

Charcot-Marie-Tooth neuropathy (CMT) is an inherited degenerative disorder of the peripheral nervous system that results in slowly progressive distal muscle weakness, atrophy and loss of proprioception in the affected areas. ${ }^{1}$ CMTX neuropathy is usually associated with mutations in exon 2 of the gap junction protein $\beta 1$ (GJB1) gene. ${ }^{1-3}$ CMT1X is caused by mutations in the GJßlgene, located on chromosome Xq13.1, that encodes the Connexin 32 (Cx32) protein. Cx32 is found in Schwann cells, oligodendrocytes, and other cell types and forms channels and hemichannels that play a role in chemical and electrical communication between cells, through transport of signaling molecules, small metabolites, and ions, and may also play a role in cell growth and resistance to cell death. ${ }^{1-3}$

The onset of symptoms in males with CMTX is usually around the second decade in life. CMTX has a more severe course in males than females. ${ }^{1-3}$ It is hypothesized that $\mathrm{X}$-linked inactivation may play a role in the range of phenotype severity in females.. ${ }^{3}$ Males are uniquely vulnerable to mutations in their single copy of X-linked genes, whereas females are often mosaic, having a mixture of cells expressing different sets of X-linked genes. ${ }^{4}$ This cellular mosaicism created by $\mathrm{X}$ inactivation in females is most often advantageous, protecting carriers of X-linked mutations from the severe clinical manifestations seen in males. ${ }^{4}$

In the above case the patient's son developed symptoms at an earlier age and severity progressed rapidly as expected in males with an X linked disorder. But the patient herself also ended up having severe symptoms though gradually unlike what is expected out of females with X linked inheritance as discussed above. This brings to the question whether the novel mutation found in the above case caused gain of function although classically CMTX is thought to be caused by a loss of function mechanism. ${ }^{4,5}$ Therefore, it is important to look for and document novel mutations to better understand the phenotypic variations and severity. ${ }^{6}$

\section{Conclusion}

CMTX has a more severe course in males compared to females due to mosaic partial inactivation of the $\mathrm{X}$ chromosome. ${ }^{3}$ But as mentioned in the above case females do develop severe disability eventually probably secondary to gain of function mutations. ${ }^{3-5}$ It is important to analyze and document these novel mutations to better understand the disease inheritance and variations in phenotype and severity. ${ }^{6}$

\section{References}

1. England JD, Gronseth GS, Franklin G, et al. Practice Parameter: evaluation of distal symmetric polyneuropathy: role of laboratory and genetic testing (an evidencebased review). Report of the American Academy of Neurology, American Association of Neuromuscular and Electrodiagnostic Medicine, and Am. Neurology. 2009;72(2):185192. doi:10.1212/01.wnl.0000336370.51010.al.

2. Murphy SM, Laura M, Fawcett K, et al. CharcotMarie-Tooth disease: frequency of genetic subtypes and guidelines for genetic testing. J Neurol Neurosurg Psychiatry. 2012;83(7):706-710. doi:10.1136/jnnp-2012-302451

3. Wang Y, Yin F. A Review of X-linked CharcotMarie-Tooth Disease. J Child Neurol. 2016;31(6):761-772. doi:10.1177/0883073815604227.

4. Migeon BR: Females Are Mosaics: X Inactivation and Sex Differences in Disease, New York, Oxford University, 2007

5. Basri R, Yabe I, Soma H, Matsushima M, Tsuji S, Sasaki H. X-linked Charcot-Marie-Tooth disease (CMTX) in a severely affected female patient with scattered lesions in cerebral white matter. Intern Med. 2007;46(13):1023-7. doi: 10.2169/internalmedicine.46.0047. Epub 2007 Jul 2. PMID: 17603245.

6. Siskind CE, Panchal S, Smith CO, et al. A review of genetic counseling for Charcot Marie Tooth disease (CMT). J Genet Couns. 2013;22(4):422-436. doi:10.1007/ sl0897-013-9584-4. 